\title{
Perioperative morbidity and mortality comparison in circumferential cervical fusion for osteomyelitis versus cervical spondylotic myelopathy
}

\author{
Rishi Wadhwa, M.D., Praveen V. Mummaneni, M.D., Darryl Lau, M.D., Hai Le, B.S., \\ Dean Chou, M.D., and Sanjay S. Dhall, M.D. \\ Department of Neurosurgery, University of California, San Francisco, California
}

\begin{abstract}
Object. The most common indications for circumferential cervical decompression and fusion are cervical spondylotic myelopathy (CSM) and cervical osteomyelitis (COM). Currently, the informed consent process prior to circumferential cervical fusion surgery is not different for these two groups of patients, as details of their diagnosis-specific risk profiles have not been quantified. The authors compared two patient cohorts with either CSM or COM treated using circumferential fusion. They sought to quantify perioperative morbidity and postoperative mortality in these two groups to assist with a diagnosis-specific informed consent process for future patients undergoing this type of surgery.

Methods. Perioperative and follow-up data from two cohorts of patients who had undergone circumferential cervical decompression and fusion were analyzed. Estimated blood loss (EBL), length of stay (LOS), perioperative complications, hospital readmission, 30-day reoperation rates, change in Nurick grade, and mortality were compared between the two groups.

Results. Twenty-two patients were in the COM cohort, and 24 were in the CSM cohort. Complications, hospital readmission, 30-day reoperation rates, EBL, and mortality were not statistically different, although patients with COM trended higher in each of these categories. There was a significantly greater $\operatorname{LOS}(\mathrm{p}<0.001)$ in the COM group and greater improvement in Nurick grade in the CSM group $(\mathrm{p}<0.001)$.

Conclusions. When advising patients undergoing circumferential fusion about perioperative risk factors, it is important for those with COM to know that they are likely to have a higher rate of complications and mortality than those with CSM who are undergoing similar surgery. Furthermore, COM patients have less neurological improvement than CSM patients after surgery. This information may be useful to surgeons and patients in providing appropriate informed consent during preoperative planning. (http://thejns.org/doi/abs/10.3171/2014.5.FOCUS14140)
\end{abstract}

\section{KEY WORDS • osteomyelitis - cervical spondylotic myelopathy • matched cohort • circumferential fusion}

$\mathrm{C}$ RCUMFERENTIAL cervical fusion is indicated for various pathologies, including deformity, myelopathy, infection, and tumor. The two most common indications for surgery are cervical spondylotic myelopathy (CSM) and cervical osteomyelitis (COM). The treatment of patients with COM can be challenging, as these patients often have additional comorbidities. We set out to compare circumferential cervical fusion in patients with $\mathrm{COM}$ versus in those with CSM to identify differences in perioperative morbidity and mortality. We hypothesized that the number of complications, hospital readmissions, and mortalities would be higher in the COM group. A comparison of COM with CSM has not been done to delineate the perioperative risk profile of these two patient groups.

\section{Methods}

The University of California, San Francisco, institutional review board on cervical pathology approved the protocol for this retrospective study. We searched our institutional database for consecutive cases of CSM and

\footnotetext{
Abbreviations used in this paper: $\mathrm{COM}=$ cervical osteomyelitis; $\mathrm{CSM}=$ cervical spondylotic myelopathy $; \mathrm{EBL}=$ estimated blood loss; LOS = length of stay; PEEK = polyetheretherketone.
}

COM treated with circumferential cervical fusion performed by two senior surgeons (P.V.M. and D.C.) during the same time period (2005-2013).

Indications for surgery for COM included pathological fractures, myelopathy, and failure of antibiotic therapy. Indications for surgery in the CSM group included myelopathy and kyphosis. In both groups, multilevel anterior corpectomy was an indication for posterior fusion. For those who had one-level anterior corpectomy, significant kyphotic deformity or osteoporosis was an indication for circumferential fusion. Indications for circumferential fusion in the CSM group for the most part included significant kyphotic deformity or poor bone stock. Indications for circumferential fusion in the COM group included kyphotic deformity and the need for anterior or posterior access to an infectious process.

We analyzed the number of levels of corpectomies, use of allograft versus autograft, and use of cages (titanium or polyetheretherketone [PEEK] cage). Furthermore, the estimated blood loss (EBL), perioperative complications, short- and long-term mortality, and change in postoperative Nurick grade were compared. Perioperative complications were analyzed, including renal failure, pneumonia, wound infection, deltoid palsy, and CSF leakage. 
The Fisher exact test was used for categorical outcomes, and the Student t-test was used for continuous outcomes. The SAS 9.3 software (SAS Institute Inc.) was used for statistical analysis.

\section{Clinical Data}

\section{Results}

Our cohort included 24 patients with CSM and 22 with COM. Patient ages ranged from 20 to 73 years old, and the average age overall was 54.1 years. The average age in the COM group was 51.9 years, versus 56.1 years in the CSM group, and the two cohorts showed no significant differences in age $(\mathrm{p}=0.171$; Table 1$)$. Most patients in the COM group were male (17 [77.3\%] of 22 patients), and most in the CSM group were female (15 [62.5\%] of 24 patients). Comorbidities included kidney disease, liver disease, chronic obstructive pulmonary disease, stroke, hypertension, diabetes mellitus, heart disease, and hyperlipidemia. Differences in these comorbidities between the two patient groups were not statistically significant except in the presence of liver disease. The number of anterior levels fused ranged between 1 and 4 , and the difference between the two patient groups was not statistically significant ( $\mathrm{p}$ $=0.287$; Table 2). However, use of an autograft and the type of cage used were significantly different between the groups ( $\mathrm{p}<0.001$ for both). The EBL was a mean of 1083.3 $\mathrm{ml}$ in the COM group and $363.8 \mathrm{ml}$ in the CSM group $(\mathrm{p}=$ $0.070)$. The length of stay (LOS) in days was significantly different between the two groups: 13.9 days for the COM group versus 8.5 days for the CSM group $(\mathrm{p}<0.001)$.

The timing of surgery in the CSM group was on average 101.13 days from the onset of symptoms. Timing of surgery in the COM group was on average 10.45 days from the onset of symptoms. The difference between these two groups was statistically significant $(p=0.011)$. The percentage of patients with improvement in their postoperative Nurick grades in the COM group was less than that in the CSM group $(9.1 \%$ vs $62.5 \%$, respectively, $\mathrm{p}<0.001$; Table 3). Among those presenting to surgery within 25 days of symptom onset, $80 \%$ of CSM patients had improved Nurick grades, whereas $9.5 \%$ of those with COM had improved Nurick grades $(\mathrm{p}<0.001)$.

Table 4 shows the infectious pathogens harbored by patients in the COM group.

\section{Follow-Up}

Patients were followed up at routine intervals in the clinic. In the event that patients were unable to return to the clinic, a telephone follow-up was performed; this type of follow-up was done in only 2 patients in the COM group and 1 in the CSM group. The length of follow-up was not statistically different between the two groups: 32.9 months for the COM group and 20.0 months for the CSM group $(p=0.058)$. The COM group tended not to return for follow-up and frequently skipped follow-up visits. We lost to follow-up 5 patients in the COM group and 2 in the CSM group.

\section{Complications}

We analyzed complications including direct surgical and perioperative systemic complications. The 30-day reoperation rates were not different between the two groups $(\mathrm{p}=0.605)$. Perioperative complications occurred in 12 of 22 patients in the COM group and 8 of 24 in the CSM group $(p=0.147)$. The 30 -day mortality rate was 0 in both groups $(\mathrm{p}=0.999)$, and the 30-day readmission rate was $9 \%$ ( 2 of 22 patients) in the COM group and 12\% (3 of 24 patients) in the CSM group ( $\mathrm{p}=0.710)$. The overall death rate at the final follow-up was higher in the COM group than in the CSM group (18.8\% vs $4.5 \%, \mathrm{p}=0.159)$. There was one case of wound infection and washout in the COM group, which did not require reinstrumentation

TABLE 1: Summary of demographics for patients with COM and CSM*

\begin{tabular}{llccc}
\hline \multicolumn{1}{c}{ Parameter } & Overall & COM Group & CSM Group & p Value \\
\hline no. of patients & 46 & 22 & 24 & 0.171 \\
average age in yrs & 54.1 & 51.9 & 56.1 & 0.007 \\
male sex (\%) & $26(56.5)$ & $17(77.3)$ & $9(37.5)$ & 0.400 \\
preop Nurick grade (\%) & & & & \\
0 or 1 & $18(39.1)$ & $10(45.5)$ & $8(33.3)$ & \\
$\geq 2$ & $28(60.9)$ & $12(54.5)$ & $16(66.7)$ & 0.321 \\
comorbidity (\%) & & & & 0.021 \\
kidney disease & $6(13)$ & $4(18.2)$ & $2(8.3)$ & 0.321 \\
liver disease & $10(21.7)$ & $8(36.4)$ & $2(8.3)$ & 0.775 \\
COPD & $6(13)$ & $4(18.2)$ & $2(8.3)$ & 0.251 \\
stroke & $7(15.2)$ & $3(13.6)$ & $4(16.7)$ & 0.361 \\
HTN & $19(41.3)$ & $11(50)$ & $8(33.3)$ & 0.893 \\
DM & $8(17.4)$ & $5(22.7)$ & $3(12.5)$ & 0.592 \\
heart disease & $8(17.4)$ & $4(18.2)$ & $4(16.7)$ & 0.246 \\
HLD & $7(15.2)$ & $4(18.2)$ & $3(12.5)$ & $15(62.5)$ \\
kyphosis (\%) & $25(54.3)$ & $10(45.5)$ & & \\
\hline
\end{tabular}

* $\mathrm{COPD}=$ chronic obstructive pulmonary disease; $\mathrm{DM}=$ diabetes mellitus; $\mathrm{HLD}=$ hyperlipidemia; $\mathrm{HTN}$ = hypertension. 


\section{Circumferential cervical fusion for COM versus CSM}

TABLE 2: Summary of perioperative details and complications

\begin{tabular}{lcccr}
\hline \multicolumn{1}{c}{ Parameter } & Overall & COM Group & CSM Group & $p$ Value \\
\hline time to surgery from symptom onset in days & 57.76 & 10.45 & 101.13 & 0.011 \\
time to surgery in days among those presenting & 6.81 & 5.43 & 9.7 & 0.137 \\
$\quad$ to surgery w/in 25 days of symptom onset* & & & & 0.287 \\
no. of anterior levels fused (\%) & & & $6(25)$ & \\
1 & $8(17.4)$ & $2(9)$ & $7(29.2)$ & $<1(45.8)$ \\
2 & $17(37.0)$ & $10(45.5)$ & $22(91.7)$ & $<0.001$ \\
$\geq 3$ & $21(45.7)$ & $10(45.5)$ & $3(12.5)$ & $<0.001$ \\
autograft (\%) & $31(67.4)$ & $9(40.9)$ & 363.8 & 0.070 \\
metal cage (\%) & $17(37.0)$ & $14(63.6)$ & 8.5 & $<0.001$ \\
EBL in ml & 633.6 & 1083.3 & $8(33.3)$ & 0.147 \\
LOS in days & 11.1 & 13.9 & $0(0)$ & 0.999 \\
periop complication (\%) & $20(43.5)$ & $12(54.5)$ & $3(12.5)$ & 0.710 \\
30-day mortality (\%) & $0(0)$ & $0(0)$ & $5(20.8)$ & 0.605 \\
30-day readmission (\%) & $5(10.9)$ & $2(9.1)$ & $5(22.7)$ & \\
30-day reop (\%) & $10(21.7)$ & & & \\
\hline
\end{tabular}

* Patients presenting for surgery within 25 days of symptom onset numbered 31 overall: 21 in the COM Group and 10 in the CSM Group.

and fusion. This patient had a PEEK cage. There were 5 reoperations in each group $(\mathrm{p}=0.605)$. Most reoperations (6 overall) were performed for wound hematoma.

\section{Discussion}

Spinal osteomyelitis makes up 1\%-7\% of osseous infections. ${ }^{13}$ The incidence of spinal infection is estimated at 1 case per 100,000 persons. ${ }^{1}$ Forty percent to $80 \%$ of cases involve Staphylococcus aureus., ${ }^{2,4}$ Early recognition and diagnosis of spinal osteomyelitis is essential to prevent kyphotic deformity and neurological injury. Prior to the advent of antibiotics, approximately $40 \%-70 \%$ of patients with spinal infections died. ${ }^{14}$ Currently, intravenous followed by oral antibiotics are generally indicated when there is no neurological compromise. Antibiotics should be started as soon as tissue is obtained for culture. ${ }^{2}$ Bracing may also be considered in this group, along with close clinical and radiographic follow-up. Surgery is indicated when there is neurological compromise, instability, or deformity of the spine and when antibiotics fail to control infection. ${ }^{5}$ The principles of surgical treatment in this subgroup of patients include removal of infected tissue, restoration of neurological function, correction of deformity, and spinal stabilization (Fig. 1). ${ }^{14}$ Surgical treatment options include anterior decompression often with corpectomy followed by cage placement and fusion and/or posterior decompression with fusion depending on the location of the purulent material. If more than 3 levels are fused anteriorly, then we typically also perform posterior fusion to increase fusion rates. There is some evidence to support titanium hardware as the least likely to harbor bacteria that can cause reinfection. In a series of 88 cases of vertebral osteomyelitis treated with titanium cages, Ruf et al. showed that none had reinfection. ${ }^{12}$ On the other hand, Mondorf et al. have shown the successful use of PEEK cages in cervical ventral fusion in spondylodiscitis. ${ }^{6}$ In vertebral osteomyelitis patients, major complications have been reported to be as high as

TABLE 3: Summary of follow-up data

\begin{tabular}{lllcr}
\hline \multicolumn{1}{c}{ Parameter } & Overall & COM & CSM Group & p Value \\
\hline $\begin{array}{l}\text { length of follow-up in mos } \\
\text { follow-up Nurick grade (\%) }\end{array}$ & 25.5 & 32.9 & 20.0 & 0.058 \\
$\quad$ or 1 & $25(54.3)$ & $9(40.9)$ & $16(66.7)$ & 0.147 \\
$\quad 22$ & $21(45.7)$ & $13(59.1)$ & $8(33.3)$ & \\
$\begin{array}{l}\text { patients w/ improved Nurick grade (\%) } \\
\quad \text { overall }\end{array}$ & $17(37.0)$ & $2(9.1)$ & $15(62.5)$ & $<0.001$ \\
$\quad$ among those presenting to surgery & $10(32.2)$ & $2(9.5)$ & $8(80.0)$ & $<0.001$ \\
$\quad$ w/in 25 days of symptom onset (\%) & & & & 0.159 \\
follow-up mortality rate & 10.5 & 18.8 & 4.5 & \\
\hline
\end{tabular}

* Patients presenting for surgery within 25 days of symptom onset numbered 31 overall: 21 in the COM Group and 10 in the CSM Group. 
TABLE 4: Infectious pathogens harbored by 22 patients in the COM group*

\begin{tabular}{lc}
\hline \multicolumn{1}{c}{ Infectious Organism } & No. Infected \\
\hline Propionibacterium acnes & 1 \\
fungus & 4 \\
MRSA & 3 \\
MSSA & 6 \\
MSSE & 1 \\
Staphylococcus epidermidis & 1 \\
Enterococcus species & 3 \\
Pseudomonas & 1 \\
group B Streptococcus & 1 \\
sterile (no growth) & 1 \\
\hline
\end{tabular}

* MRSA = methicillin-resistant Staphylococcus aureus; MSSA = methicillin-sensitive S. aureus; MSSE = methicillin-sensitive Staphylococcus epidermidis.

33\%; and death rates, as high as 33\%.,3-11 Compared with thoracic and lumbar osteomyelitis, COM is rare..$^{5}$ In this study we present one of the largest groups with COM (22 patients) thus far.

Morbidity and mortality are often high in the COM group of patients, as many are plagued with bacteremia, end-stage renal disease, immunocompromised status, and intravenous drug abuse.

Among patients who had undergone circumferential fusions, we set out to compare perioperative morbidity and mortality in those with CSM and those with COM. Our goal was to generate data that could be used in the informed consent process for patients with CSM (Fig. 2) or COM undergoing cervical surgeries to give a more targeted assessment of risks for different populations undergoing similar surgeries.

Our results show that our groups of consecutive CSM and COM patients were well matched except for sex and preoperative liver disease (cirrhosis). There were no significant differences in the number of levels fused. The type of cage used for COM versus CSM was different, as we tended to use titanium in infected cases, as suggested by Ruf et al. ${ }^{12}$

Although EBL was not significantly different between the groups, there was a noticeable trend for greater EBL in the COM group versus that in the CSM group (1083.3 vs $363.8 \mathrm{ml}$ ). We think that this is because patients with $\mathrm{COM}$ are more prone to hemorrhage due to friable and inflamed tissue. Perioperative complications were higher in the COM group as well; however, the difference between the two groups was not statistically significant. The 30-day readmission was not significantly different between the groups. The LOS was significantly longer for the COM group, which can probably be explained by the need to place a tunneled intravenous line and transfer the patient to a skilled nursing facility. Interestingly, the overall death rate at the final follow-up was trending higher in the COM group (18.8\% vs $4.5 \%$ in the CSM group, $p=0.159)$. Neurological improvement in the COM patients was statistically less significant than for CSM patients.

The major study limitation was sample size. Circumferential cervical fusions are relatively rare. Note also that 5 patients in the COM group and 2 in the CSM group were lost to follow-up after hospital discharge. Unfortunately, this study may have been too underpowered to show significant differences in perioperative morbidity and mortality.

\section{Conclusions}

Circumferential cervical fusion in patients with COM has trends for higher morbidity and mortality as compared with those in patients who underwent similar procedures to treat CSM. Furthermore, the COM patients had less neurological recovery than the CSM patients. This information may be useful in the informed consent process for both groups of patients.

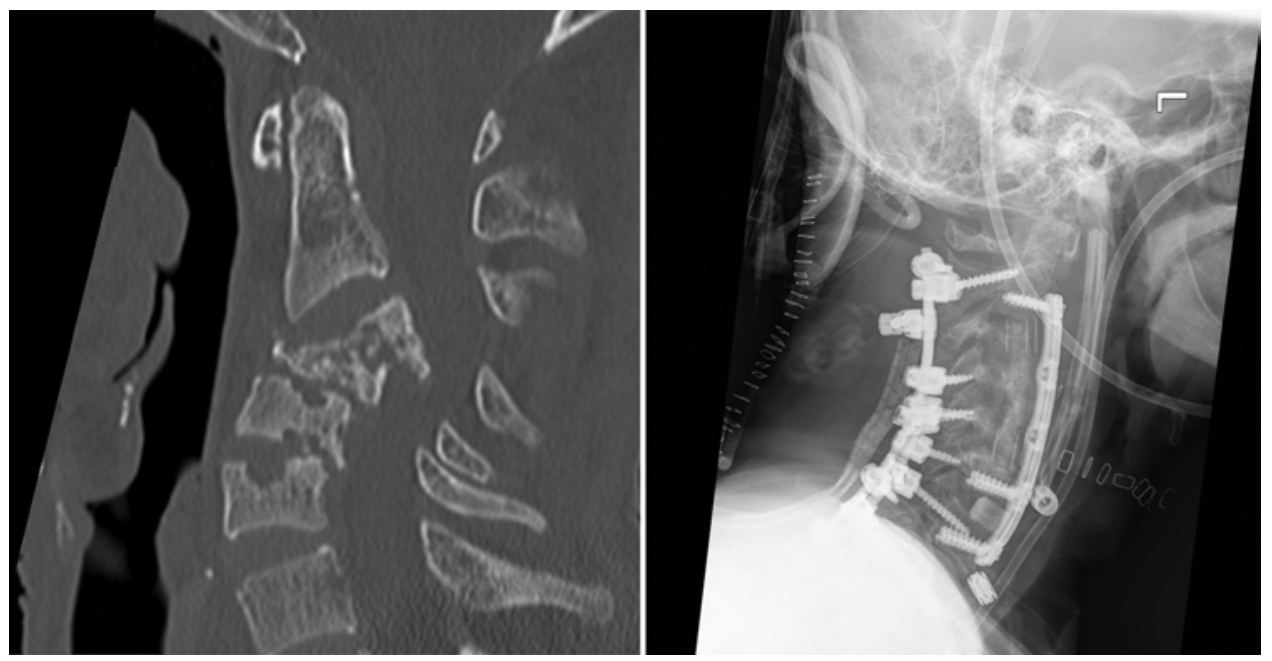

FIG. 1. Images obtained in a 67-year-old man with a 2-week history of neck pain and bilateral upper-extremity weakness. Left: Computed tomography scan shows kyphotic deformity and severe canal compromise. Right: Radiograph obtained after the patient underwent C3-5 corpectomies with C2-6 anterior fusion and evacuation of epidural abscess. He secondarily underwent a C2-T1 posterior fusion. His Nurick grade did not improve. 


\section{Circumferential cervical fusion for COM versus CSM}
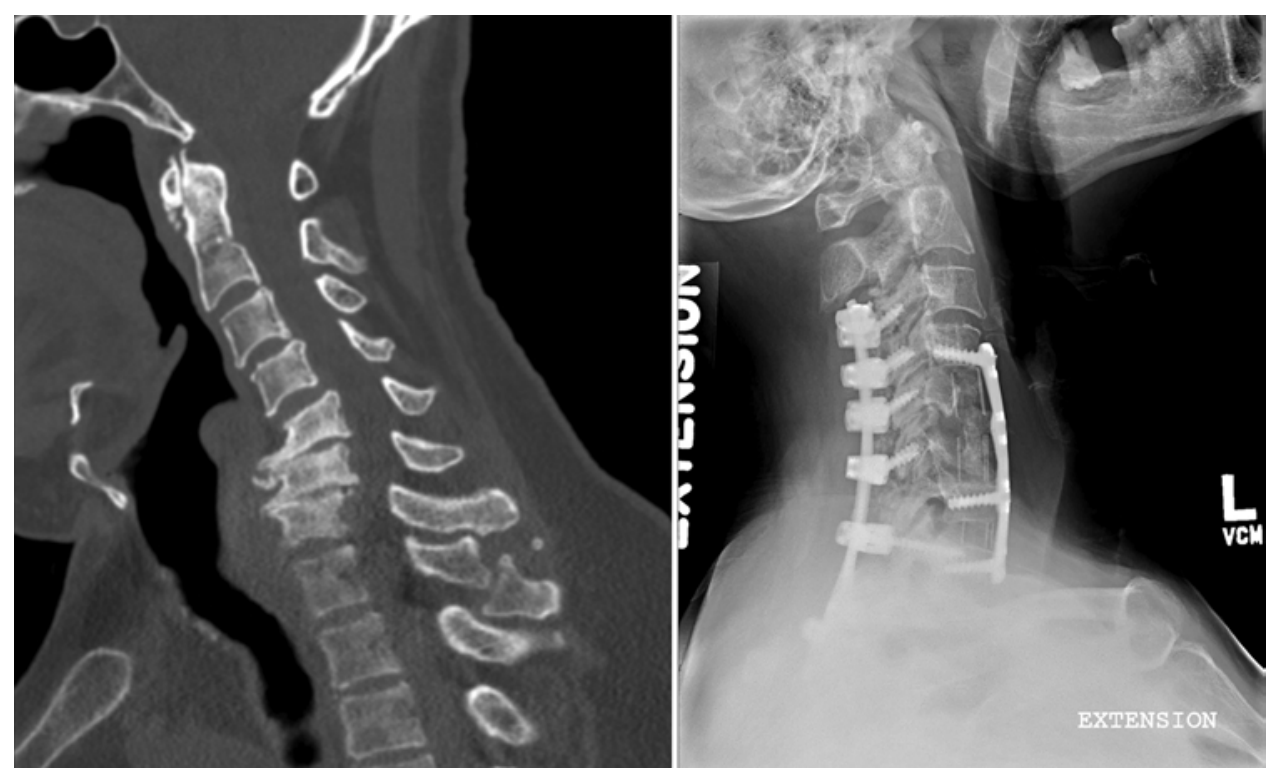

FIG. 2. Images obtained in a 58-year-old woman who had presented with neck pain and cervical myelopathy. Left: Preoperative MR image shows cervical kyphotic deformity with central canal stenosis. She underwent a C-5 corpectomy, C6-7 anterior cervical discectomy, and fusion with C4-7 fusion followed by a C3-T2 posterior fusion. Right: Radiograph shows cervical fusion at the 2-year follow-up. The patient's Nurick grade improved.

\section{Disclosure}

Dr. Mummaneni has direct stock ownership in Spinicity, receives honoraria from DePuy Spine and Globus, and receives royalties from DePuy Spine, Thieme Publishing, and Quality Medical Publishers. Dr. Chou is a consultant for Globus, DePuy Spine, Orthofix, and Medtronic. The authors report no conflict of interest concerning the materials or methods used in this study or the findings specified in this paper.

Author contributions to the study and manuscript preparation include the following. Conception and design: Wadhwa, Mummaneni. Acquisition of data: Wadhwa, Lau, Le, Chou. Analysis and interpretation of data: Wadhwa. Drafting the article: Wadhwa, Mummaneni. Critically revising the article: Wadhwa, Mummaneni. Reviewed submitted version of manuscript: Mummaneni, Dhall. Statistical analysis: Lau, Le.

\section{References}

1. Dimar JR, Carreon LY, Glassman SD, Campbell MJ, Hartman MJ, Johnson JR: Treatment of pyogenic vertebral osteomyelitis with anterior debridement and fusion followed by delayed posterior spinal fusion. Spine (Phila Pa 1976) 29:326-332, 2004

2. Hadjipavlou AG, Mader JT, Necessary JT, Muffoletto AJ: Hematogenous pyogenic spinal infections and their surgical management. Spine (Phila Pa 1976) 25:1668-1679, 2000

3. Hee HT, Majd ME, Holt RT, Pienkowski D: Better treatment of vertebral osteomyelitis using posterior stabilization and titanium mesh cages. J Spinal Disord Tech 15:149-156, 2002

4. Jensen AG, Espersen F, Skinhøj P, Rosdahl VT, FrimodtMøller N: Increasing frequency of vertebral osteomyelitis following Staphylococcus aureus bacteraemia in Denmark 19801990. J Infect 34:113-118, 1997

5. Lu DC, Wang V, Chou D: The use of allograft or autograft and expandable titanium cages for the treatment of vertebral osteomyelitis. Neurosurgery 64:122-130, 2009

6. Mondorf Y, Gaab MR, Oertel JMK: PEEK cage cervical ventral fusion in spondylodiscitis. Acta Neurochir (Wien) 151: 1537-1541, 2009
7. Mummaneni PV, Dhall SS, Rodts GE, Haid RW: Circumferential fusion for cervical kyphotic deformity. Clinical article. J Neurosurg Spine 9:515-521, 2008

8. Ogden AT, Kaiser MG: Single-stage debridement and instrumentation for pyogenic spinal infections. Neurosurg Focus 17(6):E5, 2004

9. Rath SA, Neff U, Schneider O, Richter HP: Neurosurgical management of thoracic and lumbar vertebral osteomyelitis and discitis in adults: a review of 43 consecutive surgically treated patients. Neurosurgery 38:926-933, 1996

10. Redfern RM, Miles J, Banks AJ, Dervin E: Stabilisation of the infected spine. J Neurol Neurosurg Psychiatry 51:803-807, 1988

11. Rezai AR, Woo HH, Errico TJ, Cooper PR: Contemporary management of spinal osteomyelitis. Neurosurgery 44:10181026, 1999

12. Ruf M, Stoltze D, Merk HR, Ames M, Harms J: Treatment of vertebral osteomyelitis by radical debridement and stabilization using titanium mesh cages. Spine (Phila Pa 1976) 32:E275E280, 2007

13. Schimmer RC, Jeanneret C, Nunley PD, Jeanneret B: Osteomyelitis of the cervical spine: a potentially dramatic disease. J Spinal Disord Tech 15:110-117, 2002

14. Shields LB, Raque GH, Glassman SD, Campbell M, Vitaz T, Harpring J, et al: Adverse effects associated with high-dose recombinant human bone morphogenetic protein-2 use in anterior cervical spine fusion. Spine (Phila Pa 1976) 31:542547,2006

Manuscript submitted April 15, 2014.

Accepted May 29, 2014.

Please include this information when citing this paper: DOI: 10.3171/2014.5.FOCUS14140.

Address correspondence to: Rishi Wadhwa, M.D., Department of Neurological Surgery, University of California, San Francisco, 505 Parnassus Ave., M780, San Francisco, CA 94143.email: wadhwar@ neurosurg.ucsf.edu. 